\title{
SHADOW IMAGES ON THE RETINA.
}

\author{
BY F. H. VERHOEFF, PH.B., M.D.
}

[From the Physiological Laboratory of Johns Hopkins University.]

In connection with the shadows thrown upon the retina by a small object, a pin for example, placed within the focal distance, I have recently noticed a striking optical phenomenon that is the exact opposite to the well-known pin-hole shadow experiment. The usual pin-hole experiment is produced by pricking a small hole in a card which is then held before the eye, but within the point of distinct vision. Under these conditions, if a pin is held between the hole, which serves as a source of light, and the eye, it casts an erect shadow upon the retina, and this shadow is projected as an inverted image of the pin.

This experiment was described and explained by Le Cat in 1740. It is possible, however, that he was not the first to describe it, for he does not, in his work, claim that the experiment was original with him. The following is a translation of his description (of Plate I., Figs. I and 2) of the phenomenon: " "Without betaking yourself to a dark room, put before your eye $D$ (Fig. 2), a black card $B$, pierced by a pin-hole, $C$; place opposite to and beyond this hole a very light body, such as a sheet of white paper, $E$, illuminated by a candle, $G$; place now a pin, $d$, before your eye, $D$; you will see with surprise the pin inverted, and on the other side of the hole, $F$. Let us see how this reversal and this transposition comes about. You understand that the images of external objects in passing through a hole (Fig. I) are inverted and are depicted thus inverted $* * *$ in the eye, $D$; it is the same with the images which pass through the single pin-hole,

${ }^{1}$ Le Cat, Traité des sens, Rouen, 1740, p. 297 (Helmholtz).

${ }^{2}$ Le Cat, Traité des sens, Paris, 1767 , p. 507. I have not been able to obtain the earlier edition referred to by Helmholtz. 

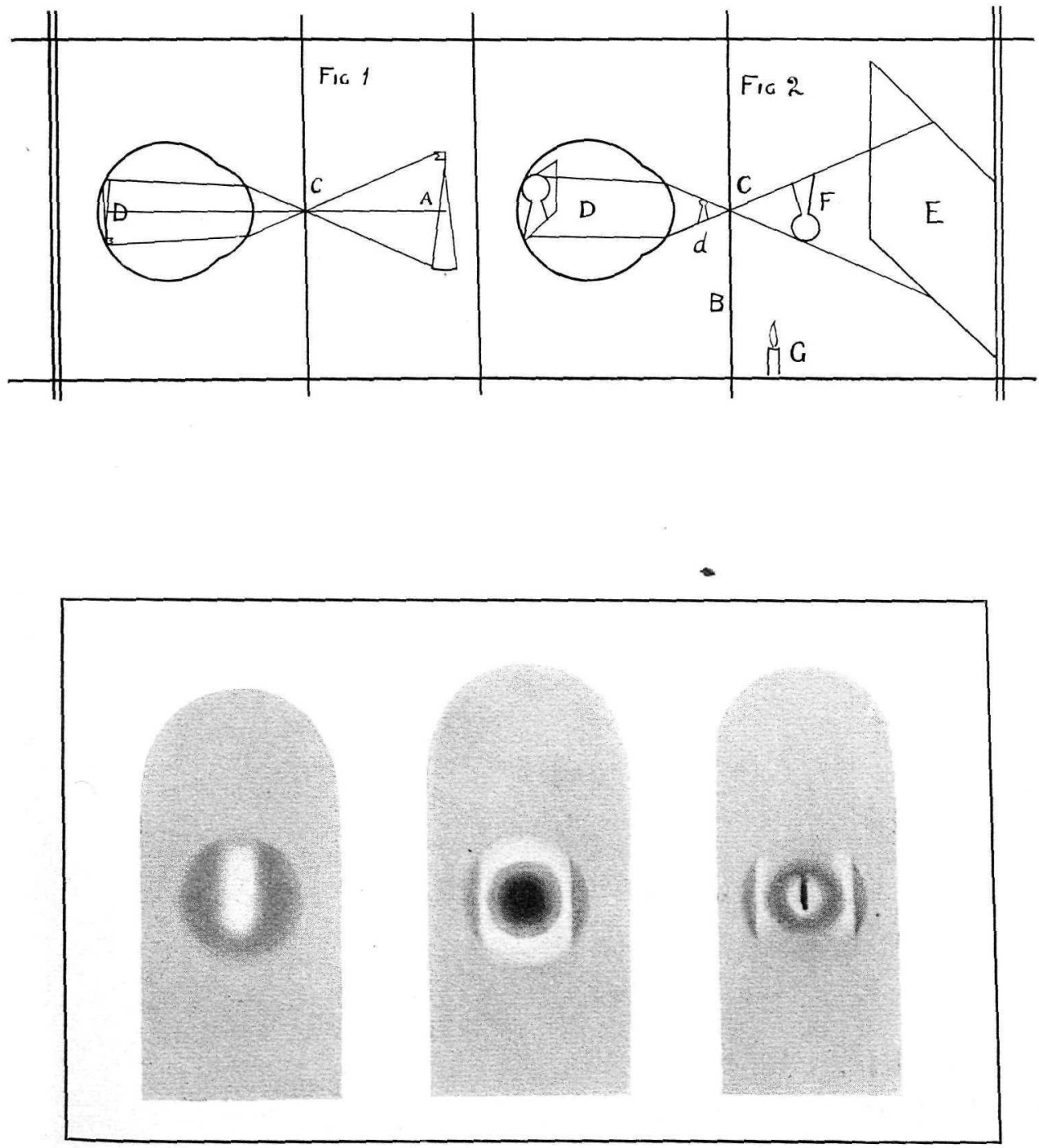

FIG. 6.

FIG. 8.

Fig. 9 .

Verhoeff on Shadow Images on the Retina. 
$C$ (Fig. 2), and which are depicted in the eye, $D$. At the spot where the upright pin, $d$, is placed, the images are already inverted; now this pin meeting these inverted images arrests the rays which correspond to it and produces consequently in these images a lack of rays, a shadow of the figure of a pin; the pin in the midst of the inverted image is upright; the image of the paper, $E$, will then be depicted at the fundus of the eye as inverted, having in its middle a shadow of the pin in a correct position; now one perceives as upright objects which are inverted in the eye and reverses those which are upright, there will be seen then the external object $E$ in an upright position and the shadow of the pin inverted; moreover, this pin, or rather this shadow of a pin, will be seen beyond the hole at $F$, because the pin which is seen is really but the shadow produced in the image of the external object $E$; this phantom pin ought then to be referred to the external object $E$, and be seen beyond the hole."

Le Cat is entirely correct in his explanation so far as he goes, but he fails to mention the importance of the position of the pin-hole with respect to the conjugate focal point of the eye, and he also fails to explain fully why it is necessary for the pin-hole to be of small size. The mention of the white sheet of paper, $E$, in the explanation is unnecessary and also misleading, since it distracts attention from the essential points involved.

J. L. Tupper, in a letter to Professor Tyndall, published in

1 Scheiner (Oculus, 1619, p. 49) described an experiment similar, in principle at least, to that of Le Cat. The following description of it is taken from 'Priestley on Vision,' Vol. I., p. 114, 1772: "If an object be suspended in a small hole made in a thin board, and an eye, situated in the dark, look through the hole at a number of torches or other luminous bodies, he observes that as many small objects will be seen as there are torches, ete. In fact, it is the shadow of the object made by each of the luminous bodies that is cast upon the eye."

In the diagram given by Scheiner the hole was represented as a comparatively large one-much larger than the pupil-and hence it could have nothing to do. with the experiment. It is essential that the torches should be out of focus, a fact evidently unknown to Scheiner. The torches act as small sources of light, corresponding to the pin-holes in La Cat's experiment, but since they are beyond the conjugate focal points of the eyes, the shadows of the objects that they throw upon the retina are inverted, and hence appear erect. This experiment should not be confused with the experiment of Scheiner referred to further on in this article. 
the Philosophical Magazine ${ }^{1}$ for 1870 , gave what he thought was the first correct explanation of the phenomenon. He was, however, evidently unacquainted with Le Cat's work on the subject. Tupper's letter was called forth by an entirely erroneous explanation given by Michael in No. 25 I of the 'English Mechanic.' Tupper states the essential explanation in a few words when he says of the experiment, 'it negatives delineation by cones and admits of it by single rays only.' This is not, of course, strictly true, since it would be impossible to obtain a hole through which only single rays could pass and still give enough illumination to make the shadow evident, but the more nearly this condition of affairs is approached the sharper is the shadow. For this reason, therefore, it is necessary that the pin-hole should be small.

Le Conte, ${ }^{2}$ in 1871 , published a short article in which he takes exception to Tupper's calling the impression of the pin on the retina an image, Le Conte insisting that it is a shadow. He says: "Now, there is no doubt that this explanation is substantially correct; but it would have been much clearer if $\mathbf{M r}$. Tupper had distinctly expressed the fact that the retinal impression in his experiment is not an image, as in ordinary vision, but a shadow. Mr. Tupper seems to have perceived the distinction but has not kept it in his mind, and hence some confusion in his deductions."

Tupper thought the experiment disproved the law of visible direction and gives the following in support of his view: "A second deduction regards the law of visible direction by which it is said an object is seen in the direction of a perpendicular to the retina at the object's point of incidence; and it is cited by its promulgator to prove the sense of vision primary and unacquired; therefore, its operation should be absolutely efficient and unindebted to experience, unconditionally indicating the direction of the object. But the law proves fallacious, the object not being in the direction of the perpendicular; it is seen where it is not. That it is seen under novel and inexperienced conditions cannot be urged without admitting experience as a

IVol. 39, Fourth Series, p. $4^{23}$

'Joreph Le Conte, Philosophical Magaxine, London, Vol. 41, p. 266. 
prerequisite to sense of direction." On this point Le Conte says : "The experiment confirms the law, as indeed does every phenomenon of vision. The law of visible direction is that every point of the retinal impression is referred to the field of vision along a line passing through that point and through the optic center. * This gives the pin inverted as we actually find it."

Le Conte was of course undoubtedly right in his statement. Tupper evidently did not have a very clear idea of what the law of visible direction is. It simply relates to the direction along which an object is apparently seen, not to the actual direction of an object, and is consistent with either theory of vision, the acquired or the congenital. The phenomenon not only confirms, as Le Conte says, but it proves the law of visible direction for it shows that no matter what may be the position of any retinal image or shadow, its apparent position is indicated by lines drawn from the image and through the optic center of the eye. As a rule of course visible direction corresponds to actual direction, but this is not necessarily true where the objects are smaller than the pupil and are out of focus.

Tupper makes another mistake when he says that a pin seen through a pin-hole is uninverted on the retina. Le Cat showed that this was not the case in his diagram (Fig. I). Le Conte also rightly says that vision through a pin-hole is in all respects similar to ordinary vision. In this connection, however, Le Conte makes a statement which is really misleading, for he says, comparing the retinal impression in pin-hole vision to that in the experiment under discussion, "the one is a true image, the other a shadow; the one is inverted, the other erect." If Le Conte meant to imply by this that the reason the latter should be called a shadow is because it is erect, he was in error, for I shall show that it depends altogether upon the position of the pin-hole with regard to the conjugate focal point of the eye as to whether the impression is inverted or even as to whether the impression occurs at all. However, the term shadow is very appropriate, although I think shadow image would be still better. Le Cat, Tupper and Le Conte seem to have entirely overlooked in their respective explanations the importance of the position of the 
pin-hole. Le Conte in his work "Sight" claims that he first explained the phenomenon and refers as proof to the article just mentioned. As a matter of fact he added little to what Le Cat and Tupper had already said.

To show the dependence of the position of the projected image upon the relative position of the pin-hole, the experiment may be modified in the following interesting way. If for instance the eye is focused for the near point and the pin-hole is held beyond this point, the shadow of an interposed pin will appear erect. Under these conditions if the pin-hole is grad-
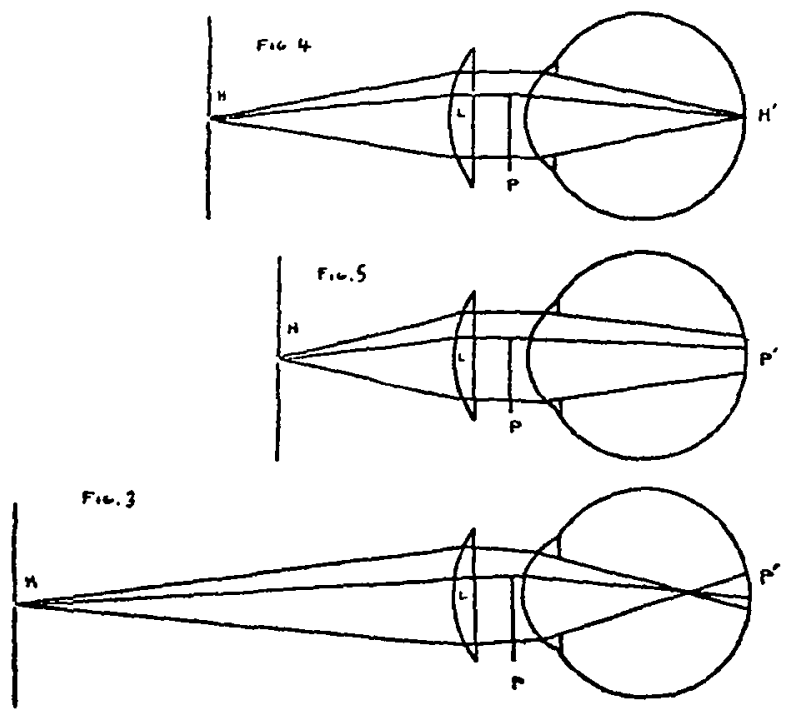

ually brought closer to the eye, the image of the pin becomes more and more blurred, and finally disappears when the hole is at the near point, that is, when the eye is accommodated for the source of light. If the hole is brought still nearer, the original experiment is reproduced, the image of the pin again appears : but is now inverted.

1 Joseph Le Conte, Sight, p. 87 , New York, 1881 . In the edition of 1897 , p. 74, Le Conte says: "This phenomenon was explained by the author in 1871 . See Philosophical Magazine, Vol. LXI., p. 266. It had, however, been previously explained by Priestley, but forgotten (Nature, Vol. XXIV., p. 80, 188I)."

I find that Priestley (Priestley on Vision, Vol. II., p, 725, London, I772), briefly summarizes Le Cat's description of the phenomenon, referring to 'Traite des Sens,' edition of i 744 , and gives all credit to Le Cat. 
This experiment is rendered easier if the accommodation is assisted by a convex lens, one of about ten diopters being very convenient. The lens is held close to the eye and the pin in front of the lens, or better immediately behind it. Then if the pin-hole be placed beyond ro $\mathrm{cm}$. from the supposedly emmetropic eye, it will appear out of focus and the shadow will be upright. As it is brought closer, the phenomenon just described takes place. The accompanying figures (Figs. 3, 4, 5) explain this experiment. In each figure, $H$ is the pin-hole, $L$ the lens, $P$ the pin. In Fig. 3, $H$ is beyond the focal distance of the lens and the shadow appears erect. In Fig. $4, H$ is in focus at $\mathrm{H}^{\prime}$ and no shadow is formed. In Fig. $5, H$ is within the focal point and the shadow appears inverted. In place of a pin-hole, a small white spot on a black surface may be used as the source of light.

I shall now describe the phenomenon referred to at the beginning of this article. If a sheet of white paper or a piece of ground glass is taken, and a small black spot is made upon it about the size of a pin-head, and this spot is used in place of the pin-hole in the above experiment, a white streak will be seen crossing the black spot (Plate I., Fig. 6). The phenomenon is best obtained without a lens, and is not an easy thing for most persons to see, since it requires that the accommodation shall be relaxed at will. I shall for convenience speak of this light streak seen crossing the black spot, as a white shadow. This white shadow behaves in exactly the same manner as does the black shadow in the pin-hole experiment-under the same conditions it may be seen upright, inverted, or be made to disappear. The experiment should be conducted in a good light, and a piece of white paper not less than five centimeters square should be used. This phenomenon may also be obtained with a photographic camera.

The explanation of the phenomenon is not quite so simple as in the case of the black shadow. The explanation suggested is illustrated in Fig. 7. This figure represents an eye accommodated for parallel rays; $A B$ is the diameter of the black spot and $P P^{\prime}$ is the cross section of the pin. The exact relative sizes of spot and pin are not represented, because the lines would 
then have to be drawn so closely together that they would not be seen clearly. Following the usual optical constructions, it will be seen that the black spot causes an area of diminished brightness, represented in section as $B^{\prime} A^{\prime}$. The lines drawn from $A B$ denote the rays which would have come from it had it the same luminosity as the rest of the visual field; the lines $B C$ and $B D$ represent the limits of the rays stopped by $B$, and the lines $A C$ and $A D$ represent the limits of the rays stopped by $A . \quad B C$ would strike the retina at $B^{\prime}$ and $A D$ would strike it at $A^{\prime}$. The continuations of $A C$ and $B D$ are not drawn, but would fall between $B^{\prime}$ and $A^{\prime}$, as would all the rays from points between $A$ and $B$. Hence $B^{\prime} A^{\prime}$ would evidently have less illumination than the rest of the field, but would still be illuminated to a certain extent, since all rays parallel to the primary axis of the eye would fall upon its center, and the rest of it would be illuminated by rays parallel to the secondary axes which strike it, of course, at every point of its surface. Now if the pin $P P^{\prime}$ is put in front of the eye, there are still more rays cut out of the general illumination, $P C I$ and $P^{\prime} D N$ denoting their limits. The pin will therefore cause a diminished brightness, extending from $I$ to $N$, and this is seen to include $B^{\prime} A^{\prime}$. But the rays included between $A P T$ and $B P^{\prime} F$ have already been cut out of the field by $A B$, and therefore $F T$ will have

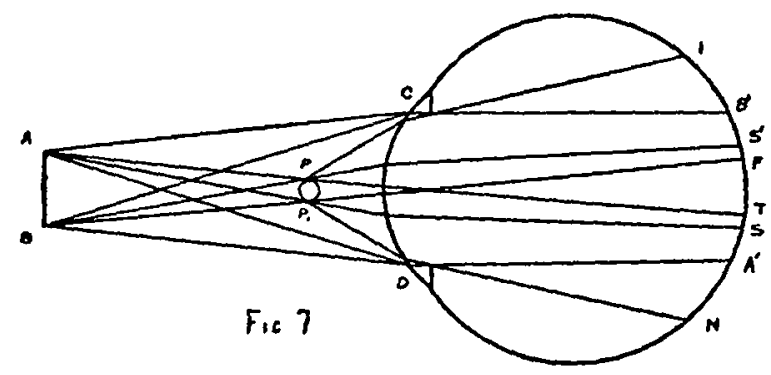

the same illumination as before, and since $\mathcal{B}^{\prime} S$ and $A^{\prime} S$ have less illumination than before the pin was placed in position, $F T$ will, by comparison, appear as a light band cutting the darkened area $B^{\prime} A^{\prime}$. In the figure, $B^{\prime} A^{\prime}$ and $F T$ represent, of course, only cross sections of areas. A figure representing 
the pin in longitudinal section may be drawn by a construction similar to Fig. 7 .

The white shadow is not absolutely clear cut because the edges shade off, owing to the fact that the original illumination of $F S^{\prime}$ and $T S$ is gradually diminished from $F$ and $T$ outward until the maximum diminution in brightness is obtain ed extending from $S^{\prime}$ to $B^{\prime}$ and from $S$ to $A^{\prime}$. Since the length of the pin is greater than the diameter of the spot, a certain number of rays which would have fallen on $F T$ are cut off and there is an actual slight diminution in the illumination of $F T$.

That physiological contrast plays a part in the perception of the white shadow is indicated by the fact that the area it occupies seems brighter than it did before the pin was brought across it, and is further shown by the fact that a red spot will give a faintly blue or green shadow. In this case, $B^{\prime} A^{\prime}$ (Fig. 7) would have an area of mixed red and white light, with the exception of $F T$, which is really white since the red rays are prevented from reaching it by the pin.

It is obvious that one of the most important factors in the formation of the shadow is the amount of illumination of $B^{\prime} A^{\prime}$, for it is the decrease in the illumination of the outer parts of $B^{\prime} A^{\prime}$ that makes the white shadow perceptible, and if the illumination is already very slight, even a total lack of light in certain parts would not be noticed. It is hardly necessary to state that the illumination must not come from the spot-the blacker the spot the better. If the black spot were in focus its image on the retina would be entirely black and the pin could not, for this reason alone, have any influence upon it, except perhaps to make the whole spot look lighter since it would diminish the brightness of the rest of the field.

If, in the place of a small spot, a sufficiently large one is used, it will be noticed that on relaxing the accommodation the center (of its image) appears much darker than the rest of the image. This is due to the fact that as the spot is made larger it cuts off more and more rays which would otherwise have reached the center of its image, and when it is as large as the pupil it cuts off all the rays parallel to the primary axis, thus producing a small spot in the center of its image totally devoid 
of light. But even when the spot is much less than half the size of the pupil the image appears darker in the center, since many rays will even then be cut off from the center of the image. This circumstance explains the fact that if a moderately large black spot is used for the shadow experiment, the white shadow of the pin is broken by a dark central spot into two white lines, somewhat resembling crescents, and giving the appearance represented in Fig. 8. If a large black spot with a small white one in the center is used, the appearance of these two crescents may be obtained, and in addition the usual black shadow is seen over the central white spot (Fig. 9).

As to the diameter of the pin which can be used the theoretical limits are from a geometrical point to a diameter equal to the distance between the lines $A C$ and $B D$, measured at the position the pin is to occupy. These do not indicate practical limits, however, for a very small pin does not cut off enough light to make sufficient difference in illumination on the retina, and in case of a large pin too much outside illumination is cut off to make the phenomenon apparent. As the diameter of the pin is increased it cuts off more and more rays from the center of $A^{\prime} B^{\prime}$, and by the use of a moderately large pin and black spot together with a convex lens I have been able to obtain a very faint, ill-defined dark line in the middle of the white shadow. It is possible, however, that this may in part be due to contrast. The size of the shadow for a fixed size and position of pin varies with the size of the spot. The distance the spot is held from the eye changes inversely as the actual diameter of both the image of the spot and the shadow, but the relative proportion of shadow to image remains constant. Other things being constant the size of the shadow varies with the distance of the pin from the eye, but not in a simple relation. The size of the shadow varies also with the size of the pin, but no matter how small the diameter of the pin its shadow can be no smaller than the diameter of the image of the spot when it is in focus. These statements apply when the eye is accommodated for parallel rays, and they seem to be borne out by practical tests.

The explanation of the erect image obtained when the spot is held beyond the conjugate focal point of the eye, and of its 
disappearance when the spot is held at this point, simply involves the crossing of the rays as in the case of the pin-hole experiment. These changes are most easily obtained with a convex lens, but it should have a diameter so large that it does not shut out any rays from the eye.

When the eye is accommodated for near points, it is no longer true that $F T$ (Fig. 7) has practically the same illumination as before the interposition of the pin, but the general principle that the illumination of $F T$ is less altered than that of $I N$ holds good. When the eye is so strongly accommodated that the pin itself is brought into focus, $F T$ becomes totally devoid of light and coincides with $I N$, which is now the sharply defined image of the pin, while the rest of the blurred image of the spot $A B$ is unaffected in any way. When the eye is accommodated for a point between the pin and the eye the white shadow again appears, since $I N$ now becomes larger than $F T$ and the illumination of the latter is diminished less than that of its immediate surroundings.

If in place of a spot a black line is used, the pin will produce a white line running down the middle of its image; the shadow in this case is more marked and more easily obtained than when a small black spot is used. Two lines drawn at a slight angle to each other will give a bend in the white shadow at their intersection, but the shadow leaves one or both of the lines at a certain distance from this point. When a narrow red line is used instead of a black one, and a lens is employed to aid the eye, the white shadow takes on a greenish or bluish color. By the use of a lens a narrow line may be so blurred as not to be seen, but a pin in front of the eye decreases so much the amount of light reaching the lateral portions of the image on the retina as to produce the appearance of a well-defined white shadow. The same thing is also true for a point.

Scheiner's experiment, in which two pin-holes placed close together are held before the eye and a double image of a pin is produced, may be explained in the same way as the white shadow just considered, since the two images obtained of the pin may be regarded as one image with a white shadow down its middle produced by the portion of the card-board between he two pin-holes. 
When simply blurring a line by relaxing the accommodation, I have noticed that if the line is not too large I can always obtain a white line running down its middle and within this line a faint dark line. In the case of a small black spot, I obtain a white spot in the center of its blurred image. I think this must be due in most part to the denser and probably less transparent nucleus of the crystalline lens shutting off more rays than the rest of the refractive apparatus of the eye. This appearance is not produced by a photographic camera and hence must be mainly due to some such peculiarity of the eye as that suggested. It is possible, however, that the positive aberration of the eye may play some part in its production. 